\title{
Spontaneous rupture of spleen in malaria: a case report
}

\author{
Sreehari $D^{1}$, Singh VB ${ }^{2}$, Meen BL ${ }^{3}$, Beniwal $S^{4}$, Ujwal $V^{5}$, Chetankumar HB $^{6}$ \\ ${ }^{1}$ Dr Sreehari Deshmukh, Post graduate student, ${ }^{2}$ Dr V B Singh, Professor and Head of Geriatric division, ${ }^{3}$ Dr Babulal Meen, \\ Assistant professor, ${ }^{4}$ Dr Sanjay Beniwal, Assistant professor, ${ }^{5} \mathrm{Dr}$ VivekUjwal, Senior resident, Senior resident, ${ }^{6} \mathrm{Dr}$ Chetan \\ Kumar HB. All affiliated with department of medicine, S P Medical College Bikaner Rajasthan, India
}

Address for correspondence: Dr Sreehari Deshmukh, Email: sreeharideshmukh@gmail.com

\begin{abstract}
Malaria is one of the most prevalent infectious diseases in the world, especially in developing world like India. Wide spectrum of complications can occur in malaria. Spontaneous rupture of spleen is one such rare complication. Here we report a case of 17 year old girl with P.Vivax malaria causing spontaneous rupture of spleen which was managed conservatively and was discharged with no complications.
\end{abstract}

Key words: Spleen, malaria, spontaneous rupture

\section{Introduction}

Malaria is endemic in India. Malaria is caused when an infected anopheles female mosquito bites humans. Causative microorganism is plasmodium species which includes P.falciparum, P.vivax, P.malaria, and P.ovale. Only falciparum and vivax is seen in India. Falciparum is notorious for causing grave complications like cerebral malaria, hepatitis, DIC. Life threatening complications with P.vivax is rare. Spontaneous rupture of spleen is extremely rare and very few cases are reported in English literature. So this problem can be overlooked especially in periphery where radiological investigations are not feasible.

\section{Case report}

17 years old girl was admitted in medicine ward of PBM hospital with history of moderate grade fever with chills and rigors for 4 days. Patient also had intense abdominal pain in left upper quadrant which was continuous in nature, dull aching, and no radiation. On admission, vitals were stable.

Patient was pale. On per abdominal examination, tender spleen was palpable 3-4 cms below left costal margin, no free fluid in peritoneal cavity, bowel sound was present. Chest auscultation was normal. Provisional diagnosis of malaria was made, blood sample was sent for routine blood chemistries. Blood chemistries showed: haemoglobin was $8 \mathrm{~g} \%$, white cell count was normal, platelet was low. Renal function and liver function tests were normal. Her ESR was $60 \mathrm{~mm}$. optimal test was positive for P.Vivax malaria by card method. Ultrasound abdomen was done which showed splenomegaly with hypo echoic regions (? splenic infarct). Coagulation profile was done which came out normal. To confirm the lesion in spleen abdominal CT with contrast was done which confirmed that the patient had spontaneous rupture of spleen.

Patient was given standard course of injectable Artesunate over 7 days followed by primaquine $15 \mathrm{mg}$ for 14 days and IV fluids and supportive treatment.Patient vitals were closely monitored. Patient was afebrile within 2 days of admission. Intensity of abdominal pain decreased after 3 to 4 days of admission. Patient was asymptomatic and discharged after 7 days.

\section{Discussion}

Malaria is known to humans since time immemorial. Malaria is prevalent in tropical countries like India. It is caused by a protozoal parasite. Patient usually presents with fever with chills and rigors and headache. Splenic enlargement is a characteristic sign of malaria.

A palpable spleen may be present within 3-4 days of the onset of symptoms and may be noted in 50-80\% of patients with malaria. ${ }^{1}$ Spleen later on becomes more hyperaemic, swollen and tender with each febrile paroxysm. With treatment spleen reduces in size usually within days to weeks. Complications with malaria are common in developing nations. The spleen appears more protective against severe manifestations of malaria in naïve than in immune subjects. ${ }^{2}$ Size of spleen indicates the parasite load and severity of infection. Splenic complications due to malaria changes in splenic structure 


\section{Case Report}

include rupture, torsion, cyst and hematoma formation, infarction, hypersplenism, ectopic spleen, and the hyper reactive malarial syndrome (previously known as tropical splenomegaly syndrome).

Rupture is more frequent in nonimmune persons than in those living in endemic areas in which multiple attacks result in gradual splenic enlargement, making rupture less likely.

Pathological rupture of the spleen occurs most commonly in malaria followed by infectious mononucleosis, kalaazar, viral hepatitis, typhoid, amyloidosis and Gaucher's disease.

There are only a couple of published studies which has estimated the incidence of spontaneous splenic rupture in malaria, rates in naturally occurring infection the range is estimated from $0 \%(0 / 5,870$ total cases of infection in Howard and colleagues series in 1973) to $2 \%$ (1/51 cases in Kahn and colleagues series in 1970) ${ }^{3}$.

Splenic complication should be considered if patient is experiencing vomiting, abdominal pain and tenderness with distension, and is having tachycardia with hypotension. Pain at the tip of the left shoulder (Kehr's sign) is an evidence of diaphragmatic irritation but it occurs in less than one half of the patients Ultrasonography of abdomen, diagnostic peritoneal lavage, arteriography, laparoscopy, CT abdomen can be used to confirm rupture of spleen. ${ }^{4}$

Exact cause for rupture in spleen is not known even though it is hypothesised that it could be due to (1) cellular hyperplasia and venous-sinusoidal engorgement leading to increased tension in the organ, (2) vascular occlusion by reticulo-endothelial hyperplasia leading to thrombosis and infarction, (3) episodic increase in the intra-abdominal pressure by activities such as sneezing, coughing and defecation, compressing the tense organ.

When acting together, these factors bring about parenchymal and sub capsular haemorrhages which strip the capsule, leading to further sub capsular haemorrhage. Eventually, the capsule tears leading to free intraperitoneal haemorrhage. ${ }^{5}$

Splenic rupture is seen usually in acute infections as in our case rather than chronic, its due to rapid hyperplasia, stretching of splenic parenchyma and capsule a high frequency of small infarctions, haemorrhage and tears, a lack of extensive connective tissue and fibrosis which is seen in chronic malarial spleens, and rapid turnover of malarial parasites.
On gross examination spleen appears dark red because of congestion, hyperaemia and deposition of haemozoin (a malarial pigment). Capsule is thin and friable. Splenic reactions to malarial infection include splenomegaly, ectopic spleen, torsion or cyst formation, haematoma formation $^{6}$, splenic infarction and rarely, abscess formation ${ }^{7}$.

Depending on the patient's condition, treatment should be decided, even though few consider splenectomy is the treatment of choice. ${ }^{8}$ Conservative management can be applied in carefully selected cases, which include strict bed rest, careful fluid management (with transfusions as required) and close clinical and haematological observations. So in this case as patient was hemodynamically stable non operative management was followed.

If operative approach is selected, spleen conserving procedures should be employed wherever possible, as spleen plays an important role in immune response to pneumococcal and malarial infection and as spleen plays an important role in maintaining immunity in a child or those living in endemic areas. ${ }^{9}$ Healing of the ruptured spleen is usually complete in 2-3 weeks time.

Splenectomy is done in patients with uncontrollable bleeding with signs of haemorrhagic shock or recurrent splenic bleeding as a last resort. ${ }^{10}$

\section{Funding: Nil}

Conflict of interest: Nil

Permission from IRB: Yes

\section{References}

1. Yagmur Y, Kara I H, Aldemir M, Büyükbayram H, TacyildizI H and Celalettin K., Spontaneous rupture of malarial spleen: two case reports and review of literature, critical care 2000, 4:309-313.

2. Buffet P A, Safeukui I, Deplaine G, Brousse V, PrendkiV, Thellier M, Turner G D., The pathogenesis of Plasmodium falciparum malaria in humans: insights from splenic physiology. The $\mathrm{J}$ of American Soc. Of haematology. 2011;117(2):381-392.

3. Howard WA, Krotoski WA, Slonim MS, Contacos PG., Spontaneous splenic rupture in Pl. vivax malaria; case reportMilitary Med 1973, 138:32-35.

4. Tauro L F, Maroli R, D’Souza R S, Hegde B R, Shetty S R, Shenoy D., Spontaneous rupture of the malarial spleen, the Saudi journal of gastroenterology; 2007 (13); 4:163-167 


\section{Case Report}

5. RabieE M,HashemeyA A, HakeemI E,Hakamy Al, ObaidM,SkainiM A,Shabbir G,SareiiS A and HussainM $\mathrm{N}$., spontaneous rupture of malarial spleen: report of two cases; Mediterr J Hematol Infect Dis,2010; vol 2(3):

6. Ozsoy MF, Oncul O, Pekkafali Z, Pahsa A, Yenen OS., Splenic complications in malaria: report of two cases from Turkey. J Med Microbiol. 2004;53:1255-8.

7. Bonnard P, Guiard JB, Develoux M, Rozenbaum W, Pialoux G., Splenic infarction during acute malaria. Trans R Soc Trop Med Hyg. 2005;99:82-6.
8. AJ Mokashi, RG Shirahatti, SK Prabhu, KR Vagholkar., Pathological rupture of malarial spleen, journal of post graduate medicine, 1992(38);3:141-2.

9. Abouzahir A, Bouchama R., Spontaneous spleen rupture in the course of malaria, MEDECINE ET MALADIES INFECTIEUSES. 2008; 38(3): 153-155

10. Zingman BS, Viner BL., Splenic complications in malaria: case report and review. Clin Infect Dis 1993, $16: 223-232$.

\section{How to cite this article?}

Sreehari D, Singh VB, Meen BL, Beniwal S, Ujwal V, Chetankumar HB. Spontaneous rupture of spleen in malaria: a case report. Int J Med Res Rev 2014;2(1):67-69, 10.17511/ijmrr.2014.i01.12 